\title{
Modelo de Interfaz Adaptativa basada en Perfiles de Usuario y Ontologías para Recomendación de Objetos de Aprendizaje
}

\author{
Thomás Quiroz, Oscar M. Salazar y Demetrio A. Ovalle \\ Universidad Nacional de Colombia, Departamento de Ciencias de la Computación y de la Decisión, \\ Facultad de Minas, Medellín-Colombia (e-mail: tquirozv@unal.edu.co, omsalazaro@unal.edu.co, \\ dovalle@unal.edu.co)
}

Recibido Ene. 23, 2018; Aceptado Mar. 21, 2018; Versión final May. 11, 2018, Publicado Dic. 2018

\begin{abstract}
Resumen
Se propone un modelo de interfaz gráfica que se adapta a las preferencias cognitivas de los usuarios y que es capaz de recomendar objetos de aprendizaje (OAs). La propuesta hace uso de la metodología Methontology la cual permite representar los conocimientos del dominio a partir de ontologías. Adicionalmente, la metodología Prometheus es utilizada para el diseño del sistema multi-agente de recomendación de OAs. La funcionalidad adaptativa consiste en distribuir los paneles que componen la interfaz según el usuario específico que esté interactuando con el sistema. La realización de esta distribución se basa en las características y preferencias de cada usuario, las cuales están almacenadas en el perfil del usuario. La validación del modelo de interfaz propuesto fue realizada mediante la aplicación de las siguientes métricas: 1) el rendimiento del sistema, (2) la experiencia de usuario, (3) la usabilidad (4) la persistencia a la sobrecarga, entre otras. Los resultados obtenidos evidencian las bondades de utilizar agentes inteligentes, ontologías y perfiles de usuario para construir interfaces de usuario adaptativas.
\end{abstract}

Palabras clave: interfaces de usuario adaptativas; ontologías; sistemas multi-agente; interfaz gráfica

\section{Adaptive Graphical User Interface based on User Profile and Ontologies to Recommend Learning Objects}

\begin{abstract}
A graphical interface model that adapts itself to the user cognitive preferences and that is able to recommend learning objects (LOs) is proposed. The proposal considers the Methontology methodology, which allows representing the knowledge of the domain based on ontologies. In addition, the Prometheus methodology is used for designing the LOs multi-agent recommendation system. The adaptive functionality consists of distributing the panels that make up the interface according to the particular user interacting with the system. The panel distribution is performed based on the characteristics and preferences of each user, which are stored in the user profile. The validation of the proposed user interface model was done by applying the following metrics: 1) system performance, (2) user experience, (3) usability (4) overload persistence, among others. The results show the benefits of using intelligent agents, ontologies, and user profiles to construct adaptive user interfaces.
\end{abstract}




\section{INTRODUCCIÓN}

Gracias a internet se ha facilitado el acceso a grandes volúmenes de información lo cual crea un problema para las personas que están en la búsqueda de contenido educativo debido a que la mayoría de la información disponible no tiene la estructura adecuada para su correcta asimilación (Acevedo et al., 2010). Como una posible solución a este problema surgen los Objetos de Aprendizaje (OAs), los cuales poseen unas características llamadas metadatos cuya función es describir en detalle y explicar la naturaleza de cada objeto. Entre los estándares más conocidos está el IEEE-LOM (Learning Technology Standards Committee, 2002) el cual describe los metadatos del objeto en varias subcategorías tales como requisitos técnicos, características pedagógicas, ciclo de vida, anotaciones (uso pedagógico del OA), clasificación, entre otros.

Debido a la gran cantidad de objetos educativos que existen en repositorios o federaciones de OAs (Zapata el at., 2013), conocidos como FROAs, es muy difícil encontrar un OA que se adecue perfectamente a los estilos de aprendizaje de las personas que lo requieren, dado que es complejo especificar completamente los criterios de búsqueda cuando se quiere acceder a un OA (Wang y Wu, 2011); los OAs no tienen el mismo efecto en toda la población debido a que cada objeto posee uno o varios estilos de aprendizaje diferentes, tales como Activo/Reflexivo, Sensitivo/Intuitivo, Inductivo/Deductivo, Visual/Verbal, Secuencial/Global (Felder y Silverman, 1988). Por todo lo anterior, existen sistemas de recomendación de OAs, con la finalidad de mejorar el acceso a los objetos digitales correctos, es decir que se adecuen a las necesidades individuales de los usuarios (Zapata et al, 2015); todo esto mediante la búsqueda en FROAs, identificando los recursos más adecuados según el perfil de cada usuario (Tipo de aprendizaje, características del estudiante y preferencias en general) que está realizando la búsqueda (Rodríguez et al., 2016). Por los anteriores motivos integrar una interfaz que facilite la adaptación del usuario al sistema de recomendación de OAs es muy importante ya que mejora y posibilita el acceso al conocimiento. Adicionalmente, las ontologías se pueden definir como un mecanismo de representación del conocimiento y así son el fundamento de la web semántica (extensión de la web actual) en donde "la información tiene un significado bien definido, es más entendible por los computadores y en donde las personas pueden trabajar cooperativa y colaborativamente" (BernersLee y Hendler, 2001). Las ontologías permiten la reutilización y accesibilidad de recursos digitales a partir de motores de búsqueda y programas computacionales los cuales realizan el filtrado y la recuperación de información. "Una ontología es el resultado de seleccionar un dominio y aplicar sobre el mismo un método para obtener una representación formal de los conceptos que contiene y las relaciones que existen entre estos" (Liang, Zhu, Tian, y Ji, 2010). Cabe señalar que las ontologías se especifican a través de lenguajes computacionales tales como OWL (por sus siglas en inglés para Web Ontology Language), con el fin de automatizar el procesamiento de la información por parte de programas basados en la web, en vez de que sea realizado en forma manual como era el caso en la Web tradicional. En el caso de las GUI, las ontologías pueden ser utilizadas para que mediante la representación de conceptos y relaciones entre ellos se pueda comprender la interacción entre los elementos de las GUI y los usuarios.

Los sistemas multi-agente (SMA) están constituidos por varias entidades (agentes), las cuales poseen una cantidad de autonomía e inteligencia suficiente como para realizar tareas específicas con poca o ninguna supervisión humana. Este tipo de sistemas autónomos se utilizan para solucionar problemas donde se requieren que varios entes trabajen individualmente y en conjunto para lograr una meta en común (Ovalle et al., 2014). Los agentes poseen dos importantes capacidades a saber: a) Acciones autónomas, lo cual implica decidir por sí mismos qué tareas necesitan hacer y en qué orden realizarlo; b) Capacidad de interacción, es decir no simplemente el intercambio de datos entre los agentes, si no la actividad social (cooperación, coordinación, negociación). Los SMA pueden ser utilizados para realizar la comunicación entre una interfaz gráfica de usuario (GUI) inteligente y un sistema de recomendación de OAs. En efecto, las GUI, han evolucionado desde interfaces eminentemente textuales a interfaces más complejas de tipo multimodal (integrando diversas formas de interacción) obteniendo como resultado que la interacción entre el ser humano y la maquina se facilite. Es importante en este ámbito explicar la diferencia entre adaptabilidad y adaptatividad en las GUI de usuario; una GUI adaptable es en la cual el usuario realiza los cambios para adecuar la interfaz a sus gustos y preferencias. Por el contrario, en una GUI adaptativa el sistema es el encargado de realizar los cambios necesarios en la interfaz con base en el perfil del usuario.

Park et al. (2007) investigan como validar la efectividad de los menús adaptativos y adaptables, para esto se estudian 3 tipos de menús, un menú adaptable, un menú adaptativo separando la posición y el último es un menú adaptativo el cual resalta las opciones del menú. Del estudio se concluye que la opción preferida por los usuarios es el menú adaptable dado que es eficiente y tiene las opciones que son preferidas por los usuarios ya que son ellos quienes realizaron los cambios. Letsu-Dake y Ntuen (2010) evalúan el impacto de las interfaces adaptativas en sistemas complejos, en los cuales el usuario vigila un proceso industrial y se deben mantener en rangos óptimos múltiples variables dentro del sistema, una interfaz adaptativa ADI (por sus siglas en inglés para Adaptive Interface) fue provista a los usuarios para monitorear el proceso. Para evaluar el efecto de la ADI se contrastó el desempeño de los usuarios que utilizaron esta interfaz contra otros 
usuarios que usaron una interfaz normal NADI (No ADI), al comparar la tasa de detección de fallas en ambos se pudo observar que los usuarios que utilizaron ADI tenían una tasa de detección y corrección de errores mayor a la de los usuarios que utilizaron NADI.

Por su parte, Jorritsma et al. (2015) estudian el efecto de una interfaz adaptativa en un entorno de trabajo, en este caso en un laboratorio médico donde la función de la GUI es visualizar imágenes de radiología (PACS). La función adaptable del sistema consiste en una pestaña llamada "My tab" ubicada en la barra de herramientas y un menú desplegable cuando se oprime el botón derecho del mouse. La funcionalidad de estas opciones es agregar las opciones del menú que el usuario utiliza comúnmente y de esa forma poder acceder a ellas más rápidamente. Ghédira et al. (2002) desarrollan una aplicación web con una interfaz adaptativa diseñada para revisar exámenes médicos de cardiología. La interfaz monitorea el uso que le dan los usuarios y de esta forma es capaz de realizar su adaptación, la cual consiste en crear un enlace genérico ("Generic link") con todas las opciones más utilizadas por dicho usuario. De esta forma el usuario se ahorra el tiempo que tendría que invertir en navegar por el menú de la aplicación.

Ravi et al. (2015) proponen una interfaz adaptativa para un curso online masivo y abierto (MOOC), cuya función es cambiar las opciones del menú disponible para los profesores que crean cursos en la plataforma. Los profesores son categorizados en los siguientes 4 grupos: (1) Usuario experto, (2) Usuario con buen conocimiento, (3) Usuario con conocimientos intermitentes y (4) usuario novato. Según el tipo de usuario que sea la interfaz se adapta para mostrar mayor o menor cantidad de opciones del menú. Adicionalmente, Shakshuki et al. (2015) proponen un SMA con el fin de recolectar información del estado de la salud de los pacientes; a esta información pueden acceder tanto los doctores como los pacientes. La funcionalidad adaptativa de este sistema consiste en cambiar la tonalidad de los colores facilitando de esta forma la visualización del contenido por parte de personas con problemas visuales tales como visión borrosa, daltonismo, entre otros. Park y Han (2011) tratan de unificar los conceptos de adaptatividad y adaptabilidad en una interfaz gráfica de usuario, para validar esto crearon 4 interfaces diferentes y se midió para cada interfaz: (1) el tiempo de adaptación del sistema, (2) el tiempo que se demoraron los usuarios para encontrar una opción específica, (3) la eficiencia percibida y (4) las preferencias sobre las interfaces. Se puede concluir que: a) La adaptabilidad es más apropiada que la adaptatividad cuando se requiere robustez en las variaciones; b) El usuario debe ser capaz de controlar la adaptatividad para proteger su privacidad.

Del análisis de los trabajos anteriores se constata la necesidad de crear e implementar una GUI adaptativa la cual mejore la interacción entre el humano y la máquina. El objetivo de este artículo es entonces proponer un modelo de interfaz gráfica que se adapte a las preferencias cognitivas de los perfiles de usuario, los cuales son formalizados a través de ontologías y de esta forma ser capaz de recomendar objetos de aprendizaje (OAs). Igualmente, se busca validar el modelo de interfaz propuesto mediante la aplicación de las siguientes métricas al prototipo construido: 1) el rendimiento del sistema, (2) la experiencia de usuario, (3) la usabilidad (4) la persistencia a la sobrecarga, entre otras, aplicadas sobre varios casos de estudio.

\section{DESARROLLO DEL MODELO}

La GUI desarrollada en este modelo (ver figura 1) se basa en agentes, los cuales son los encargados de realizar las adaptaciones de la interfaz (e.g. cambiando la posición y/o los tamaños de los paneles que posee la interfaz) teniendo en cuenta los datos almacenados en el perfil de cada usuario.

La interfaz está compuesta de cinco paneles, cada panel tiene una función única: 1) Panel de Menú: En este panel se agrupan las diferentes opciones de menú para realizar algunas tareas como: Buscar OA o desplegar un OA recomendado; 2) Panel de búsqueda: La función de este panel es realizar búsquedas simples de OAs; 3) Panel de contenido: Este panel está enfocado en mostrar los OAs que el usuario ha seleccionado; 4) Panel de notas: Este panel fue diseñado para que el estudiante pueda tomar apuntes de lo que se ha aprendido mientras estudia el contenido de los OAs; y 5) Panel de repositorios: La función de este panel es permitir un acceso rápido a diferentes repositorios, permitiendo a los estudiantes acceso a otros OAs que no hay en el sistema.

Los perfiles de usuario son creados a partir de un grupo de información específica de una persona que permiten definir los gustos y preferencias del usuario. De esta forma se puede predecir sus intenciones (Alaoui et al., 2015). La base para la recomendación de OAs, es el perfil del usuario, el cual contiene las características personales, preferencias, gustos, historial de búsquedas, estilo de aprendizaje entre otros. Para poder organizar toda esta información es necesario tener un modelo de perfil de usuario. El perfil de usuario consta de los siguientes 5 elementos: datos personales, estilo de aprendizaje, historial, GUI y preferencias. Estos datos del perfil de usuario son capturados cuando una persona entra por primera vez al sistema, debe de llenar unos campos con información personal y responder un cuestionario con el fin de obtener los datos del estilo de aprendizaje de la persona, todo esto para poder crear su usuario dentro del sistema. 


\section{User: Thomas Quiroz}

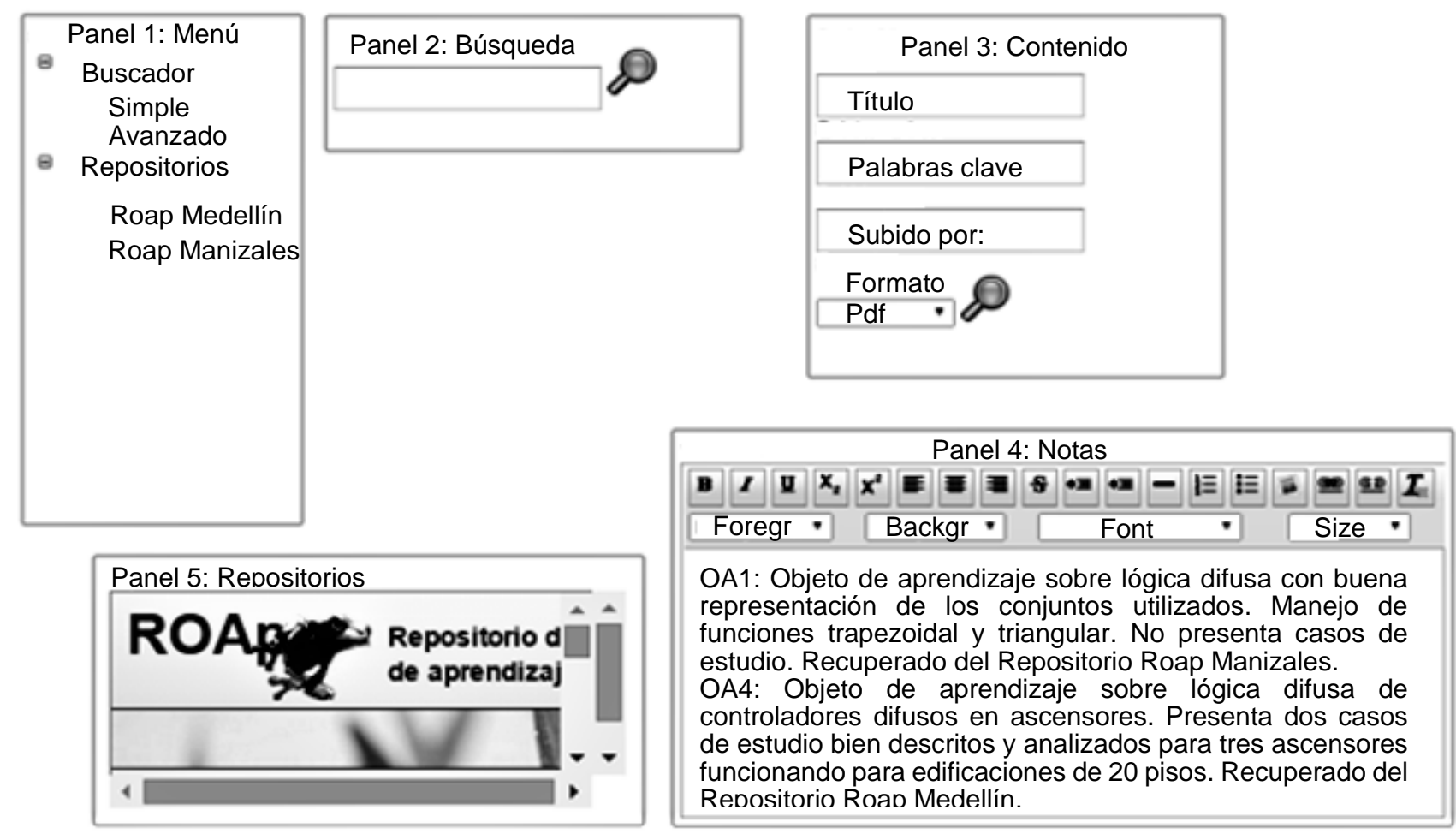

Fig. 1: GUI del modelo propuesto. Adaptada de (Quiroz, 2017)

Las ontologías proporcionan una gama de herramientas que permiten representar el conocimiento (Salazar, 2015), en este caso es utilizada para poder inferir cual es la distribución propicia de páneles en la GUI para un usuario específico. La metodología seleccionada para crear la ontología fue Methontology (FernándezLópez et al, 1997), la cual proporciona las guías para el desarrollo e implementación de la ontología a través de las siguientes etapas: (1) fase de especificación, (2) fase de conceptualización, (3) fase de formalización, (4) fase de implementación y (5) fase de mantenimiento. La figura 2 muestra la ontología generada a partir del conocimiento identificado durante el desarrollo de las fases mencionadas anteriormente, a través de un lenguaje formal, en este caso se utilizó OWL (Ontology Web Language) mediante la herramienta Protégé (Tudorache et al, 2013).

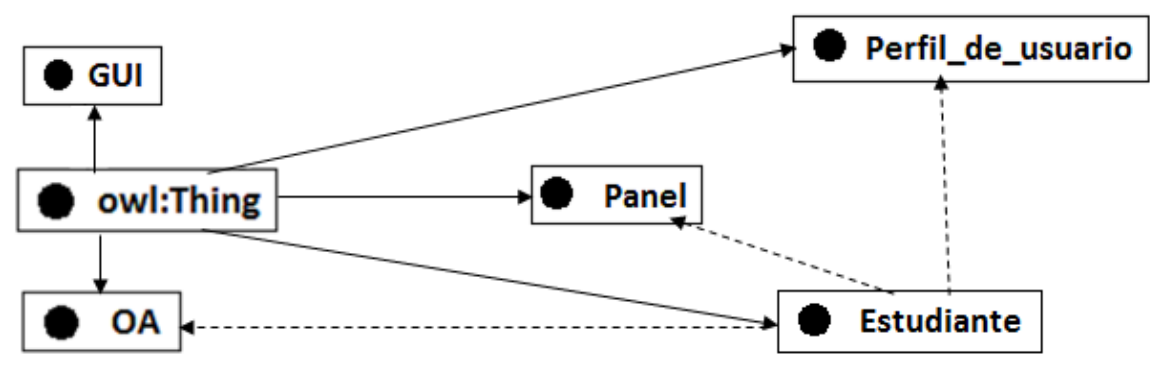

Fig. 2: Ontología generada a partir de Protégé. Adaptada de (Quiroz, 2017)

Los sistemas de recomendación (SR) de OAs ayudan a los usuarios a encontrar recursos educativos adicionales y al mismo tiempo relevantes la búsqueda que se está realizando (Park et al., 2012). En entornos de e-learning los SR tienen la función de resolver el problema de la sobrecarga de información al mostrarle al usuario otros OAs que se adecuen a sus necesidades (Sanjuán et al., 2009).

EI SR del modelo propuesto tiene en cuenta todos los perfiles de los usuarios que existen en el sistema con base en esto es posible entregar más y mejores recomendaciones. Lo anterior es posible gracias a que el sistema de recomendación del modelo creado posee 3 tipos diferentes de filtrado: (i) Filtrado colaborativo: Las recomendaciones basadas en este tipo de filtrado, entregan OAs que otros usuarios con un perfil similar al del usuario activo han visualizado; (ii) Filtrado basado en conocimiento: Este tipo de filtrado hace recomendaciones basándose en OAs similares a los que el usuario seleccionó en el pasado; y (iii) Filtrado basado en contenido: Este tipo de recomendación está basado en el perfil del usuario, basándose en el estilo de aprendizaje del usuario que realiza la búsqueda. 


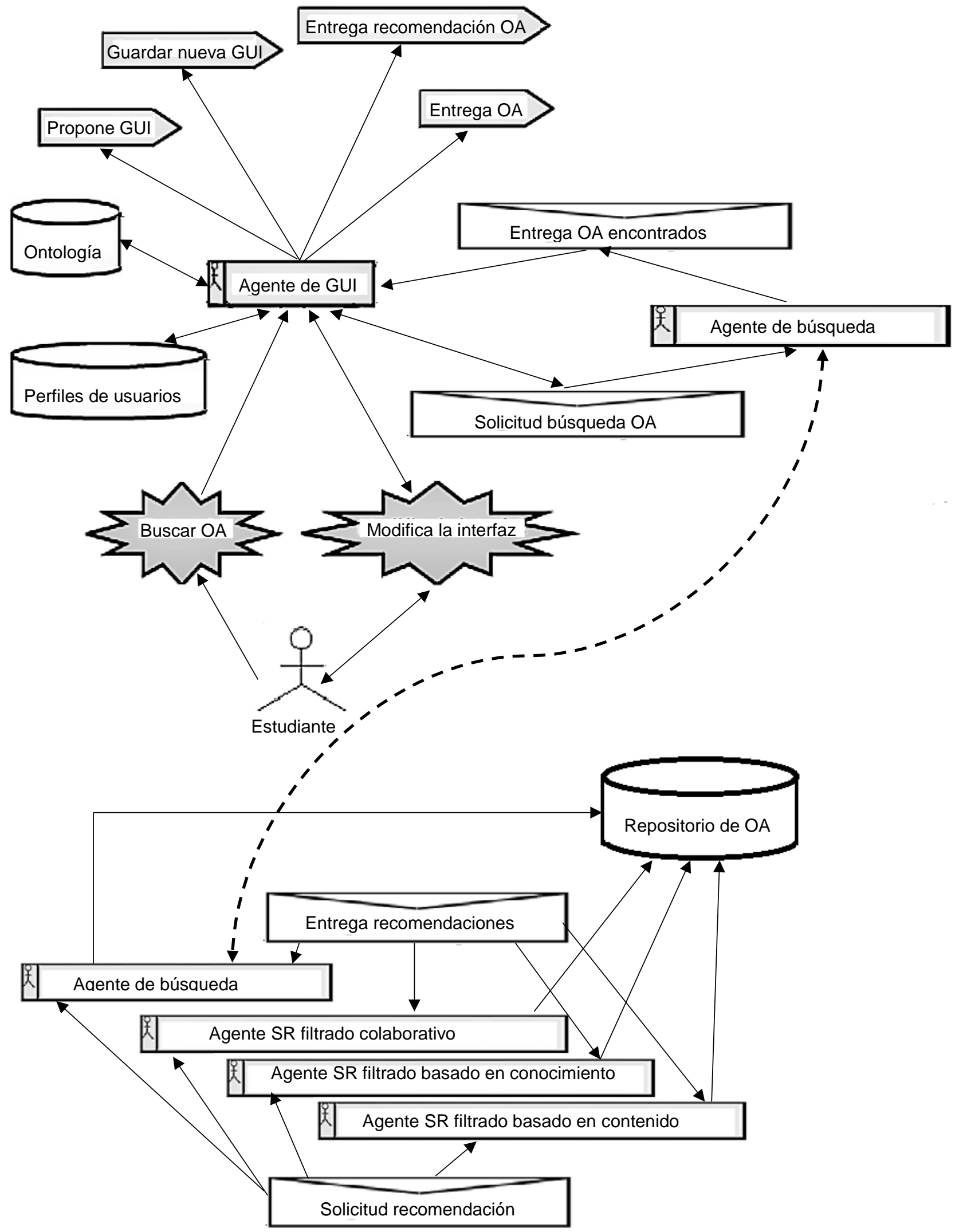

Fig. 3: Vista general del SMA. Adaptada de (Quiroz, 2017).

Para diseñar el SMA se utilizó la metodología Prometheus (Lhafiane et al., 2015) la cual está basada en el paradigma orientado a agentes y para el desarrollo del SMA se utilizó la herramienta Prometheus Design Tool (PDT). La figura 3 presenta el diseño general del SMA, generado por la herramienta PDT, en donde se observa el flujo de información y el actor "Estudiante" a partir del cual se activa la ejecución del sistema. El SMA diseñado lo componen varios agentes, cada uno de ellos posee una función específica dentro del sistema: 
Agente de GUI: Agente de búsqueda: Agente SR Filtrado Colaborativo: Agente SR Filtrado Conocimiento: Agente SR Filtrado Contenido.

Agente de GUI: Este agente tiene como funcionalidad guardar los cambios que el usuario activo en el sistema realiza sobre la GUI. Adicionalmente, realiza la adaptación de la GUI cuando el usuario selecciona un OA y finalmente, notifica al agente de búsqueda cuando el usuario realiza una búsqueda.

Agente de búsqueda: Este agente se encarga de realizar la búsqueda de los OAs que el usuario ingresa en la barra de búsquedas. Adicionalmente, notifica a los agentes de SR de la búsqueda realizada, para que estos puedan entregar las recomendaciones pertinentes.

Agente SR Filtrado Colaborativo: Este agente entrega las recomendaciones basadas en filtrado colaborativo.

Agente SR Filtrado Conocimiento: Este agente entrega las recomendaciones basadas en filtrado por conocimiento.

Agente SR Filtrado Contenido: Este agente entrega las recomendaciones basadas en filtrado por contenido.

\section{EXPERIMENTACIÓN Y RESULTADOS}

La validación del modelo se llevó a cabo mediante la implementación de un prototipo basado en el modelo propuesto. La figura 4 presenta el diagrama de despliegue de los componentes de la arquitectura del sistema conformado por dos módulos: a) Servidor Windows, que es el módulo en el cual se despliega la base de datos, el aplicativo web y la plataforma central del sistema multi-agente y b) el dispositivo cliente, el cual permite acceder a la página web del aplicativo y el agente de GUI. Este último fue construido basado en el lenguaje GWT (Google Web Toolkit) (Tacy et al, 2013) el cual está basado en el lenguaje de programación orientado a objetos JAVA cuyo compilador lo traducirá a HTML y JavaScript. Lo anterior facilita el desarrollo de todo el prototipo dado que los diferentes elementos que compondrán el aplicativo utilizan lenguajes basados en JAVA. Por su parte el sistema multi-agente desarrollado fue implementado bajo el Framework JADE (Spanoudakis y Moraitis, 2013), el cual está desarrollado totalmente en JAVA, lo que simplifica su implementación. Cabe señalar que un sistema basado en JADE puede ser distribuido en diferentes máquinas y su configuración puede ser controlada remotamente todo esto mediante interfaces gráficas. A dicho prototipo se le evaluó su desempeño a través de métricas con el fin de validar las características más importantes del sistema. Las seis métricas consideradas son las siguientes: a) Rendimiento del sistema; b) Experiencia del usuario con respecto a las recomendaciones de GUI; c) Experiencia del usuario con respecto a las recomendaciones de OAs; d) Comportamiento del prototipo en los navegadores; e) Usabilidad; f) Grado de resistencia a la sobrecarga.

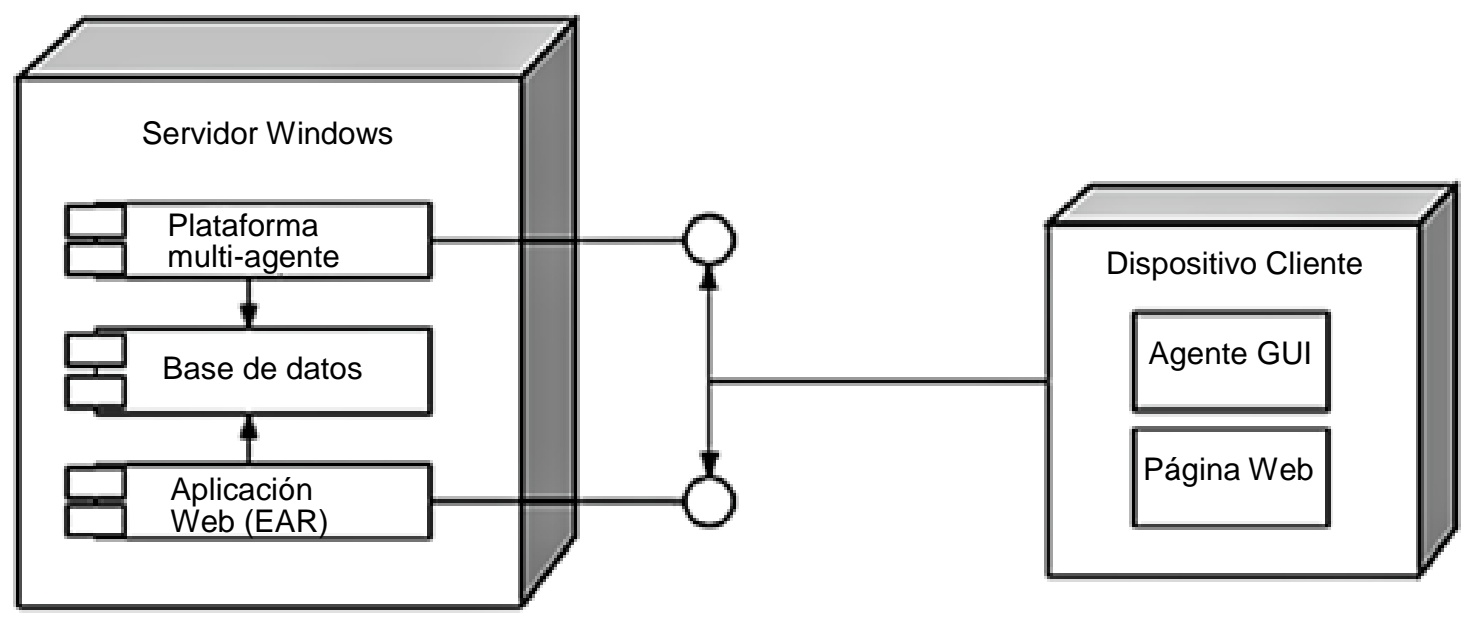

Fig. 4: Diagrama de Despliegue de los componentes de la arquitectura del sistema. Adaptada de (Quiroz, 2017).

Para realizar la experimentación se seleccionaron 20 ingenieros de sistemas (5 mujeres y 15 hombres). Cada uno de ellos tuvo que registrarse en el sistema, mediante el diligenciamiento de un formulario de registro con el fin de crear su perfil de usuario, el cual contiene los datos personales tales como: cedula, nombre, apellidos, teléfono, dirección, entre otros. Además de los datos anteriores en esta pantalla también se realizan una serie de preguntas, en total 24 basadas en el test de Felder y Silverman para obtener el estilo de aprendizaje específico (Activo/Reflexivo, Sensitivo/Intuitivo, Inductivo/Deductivo, Visual/Verbal, Secuencial/Global). El procedimiento que se siguió en los experimentos para la validación de las recomendaciones de la interfaz adaptativa consistió en dividir los 20 usuarios en 3 grupos de estudio de la siguiente forma: a) Cuatro usuarios a los cuales nunca se les activa la funcionalidad adaptativa de la GUI; b) Ocho usuarios a los cuales se 
evaluará primero sin la funcionalidad adaptativa y luego se les activa esta funcionalidad y finalmente c) Ocho usuarios con la funcionalidad adaptativa en todo momento.

Validación de la Métrica "Rendimiento del sistema"

Para aplicar la métrica del rendimiento del sistema se tomó como muestra inicial el tiempo que cada usuario invirtió en modificar la interfaz inicial del sistema según sus preferencias, los datos obtenidos fueron los siguientes: (1) promedio $76.5 \mathrm{seg}$, (2) mínimo $21 \mathrm{seg}$ y (3) máximo $154 \mathrm{seg}$. Luego se les pidió a 12 usuarios que no contaban con la funcionalidad adaptativa del sistema, modificar la interfaz inicial del sistema, los resultados obtenidos por este grupo fueron los siguientes: El promedio del tiempo empleado en la modificación de la interfaz gráfica fue de 65.9 segundos, con un valor mínimo de 3 segundos y un valor máximo de 170 segundos. A continuación, se realizó el mismo ejercicio con 16 usuarios los cuales si tenían la funcionalidad adaptativa del sistema y los datos obtenidos fueron los siguientes: El promedio del tiempo empleado en la modificación de la interfaz gráfica fue de 14.3 segundos, con un valor mínimo de 0 segundos y un valor máximo de 71 segundos. Se puede ver que la métrica de rendimiento produce resultados satisfactorios ya que para la mayoría de los formatos de los OAs seleccionados los usuarios aceptaron la distribución que el sistema propone, además se logra disminuir los tiempos de modificación de la GUI (característica de adaptabilidad) por parte de aquellos usuarios que no aceptaron la recomendación ofrecida por la interfaz adaptativa.

Las recomendaciones del sistema fueron idóneas

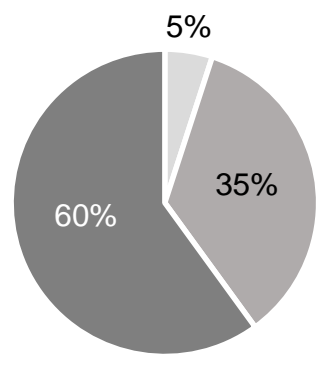

$$
-3-4=5
$$

La ubicación de las recomendaciones es adecuada

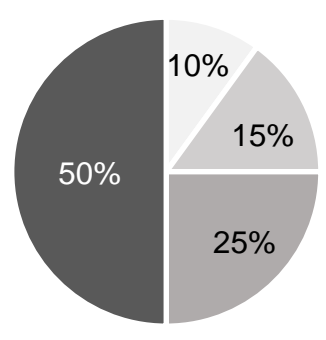

$$
2-3-4-5
$$

La cantidad de OAs recomendados es adecuada

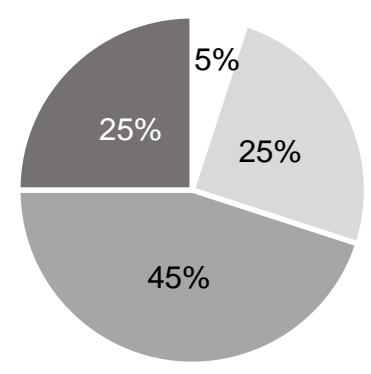

$2-3=4-5$

La calificación del SR

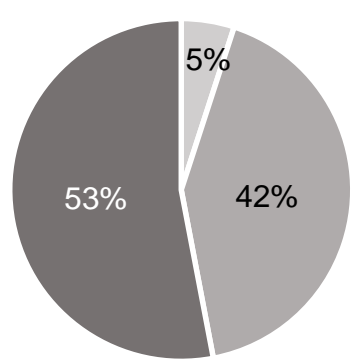

$-3-4=5$

Fig. 5: Encuesta sobre OAs recomendados por el SR. Adaptada de (Quiroz, 2017).

\section{Validación Métrica "Experiencia del usuario con respecto a las recomendaciones de GUI"}

Para validar esta métrica se tomaron datos de usuarios que utilizaron el sistema sin la funcionalidad adaptativa y con esta, estos datos se dividieron según el tipo de formato del OA que se estaba visualizando y se obtuvieron los siguientes porcentajes de aceptación: Video=56.3\%; PDF=62.5\%; Imagen=87.5\%. Al analizar los resultados se puede concluir que la función adaptativa del sistema logra disminuir los tiempos que se invierten en la modificación de la interfaz por parte de los usuarios. Lo anterior debido a que la interfaz adaptativa realizó propuestas de distribución de los páneles, basado en el histórico de distribuciones realizadas por los usuarios y otros usuarios con un perfil de usuario similar. 


\section{Validación Métrica "Experiencia del usuario con respecto a las recomendaciones de OAs"}

La validación de esta métrica se realizó mediante 2 pruebas: (1) se realizó a los usuarios una encuesta de percepción y (2) con 20 OAs de 4 temáticas diferentes se validó si el sistema entregaba el número de recomendaciones correcta. En la figura 5 se pueden observar los resultados de la encuesta realizada, Las respuestas de la encuesta tenían un valor de 1 a 5 , siendo 1 la peor calificación y 5 la mejor. La tabla 1 presenta los resultados de las recomendaciones realizadas por el SR y cuál es el porcentaje de acierto del sistema. Para este caso de estudio se tuvieron en cuenta 20 objetos de aprendizaje en el área de sistemas y computación los cuales estaban agrupados en 4 categorías (ver columna 1, tabla 1) a saber: (1) análisis y diseño de sistemas de información, (2) auditoria de sistemas, (3) base de datos y (4) Lenguajes de programación. Se puede observar según los resultados de la tabla 1 que las recomendaciones del SR por los diferentes filtrados (Colaborativo, Conocimiento y contenido) tienen un alto porcentaje de acierto.

Tabla 1: Porcentaje de acierto del SR en las recomendaciones. Tomada de (Quiroz, 2017).

\begin{tabular}{|c|c|c|c|}
\hline Categoría & OAs recomendados & $\begin{array}{c}\text { OAs que se debieron de } \\
\text { recomendar }\end{array}$ & $\begin{array}{c}\text { Porcentaje de } \\
\text { acierto }\end{array}$ \\
\hline 1 & 3 & 6 & $50 \%$ \\
\hline 2 & 6 & 8 & $75 \%$ \\
\hline 3 & 2 & 2 & $100 \%$ \\
\hline 4 & 3 & 5 & $60 \%$ \\
\hline
\end{tabular}

Validación métrica de comportamiento del prototipo en los navegadores

Tabla 2: Validación del sistema en los navegadores. Tomada de (Quiroz, 2017).

\begin{tabular}{|l|c|c|c|c|}
\hline \multicolumn{1}{|c|}{ Funcionalidad } & Chrome & Firefox & Edge & Safari \\
\hline $\begin{array}{l}\text { ¿Se visualiza correctamente todos los } \\
\text { elementos de la GUI? }\end{array}$ & si & no & si \\
\hline $\begin{array}{l}\text { ¿La funcionalidad adaptable de la GUI } \\
\text { funciona correctamente? }\end{array}$ & si & si & si & si \\
\hline $\begin{array}{l}\text { ¿La funcionalidad adaptativa de la GUI } \\
\text { funciona correctamente? }\end{array}$ & si & si & no & no \\
\hline $\begin{array}{l}\text { ¿Los OAs con formato video se } \\
\text { visualizan correctamente? }\end{array}$ & no & si & no & si \\
\hline $\begin{array}{l}\text { ¿Los OAs con formato PDF se visualizan } \\
\text { correctamente? }\end{array}$ & si & si & no & si \\
\hline $\begin{array}{l}\text { ¿Los OAs con formato imagen se } \\
\text { visualizan correctamente? }\end{array}$ & si & si & si & si \\
\hline
\end{tabular}

El prototipo realizado se validó con 4 de los exploradores más utilizados a nivel mundial a saber: Chrome, Firefox, Edge y Safari, con la intención de verificar si todos los componentes se visualizan correctamente, también se verificó que todas las características funcionaran correctamente. En la tabla 2 se puede observar todos los criterios con los que se realizó la evaluación del sistema en los navegadores. Los resultados de esta evaluación fueron bastante positivos dado que en todos los navegadores la funcionalidad adaptativa y adaptable del prototipo funcionó correctamente. Además, la mayoría de los formatos con los que se realizaron los casos de prueba se visualizan correctamente en todos los exploradores, en el caso del formato video en los navegadores Chrome y Safari, se descarga el archivo del video al computador del usuario por lo cual el video no se visualiza mediante el prototipo; en el caso del navegador Edge al tratar de visualizar el video aparece un error en el panel de contenido y tampoco se descarga el video. En la visualización de los componentes, el único navegador que presenta algún defecto es Firefox ya que este no muestra el icono que aparece en la esquina superior izquierda en la mayoría de las pantallas.

\section{Validación métrica de usabilidad}

Para validar la métrica de Usabilidad de la Interfaz se le preguntó a los usuarios del sistema sobre 4 de los páneles que componen la interfaz a saber: Menú, Búsquedas, Repositorio y Notas (No se preguntó por el panel de contenido), las respuestas de la encuesta tenían un valor de 1 a 5 , siendo 1 la peor calificación y 5 la mejor. 

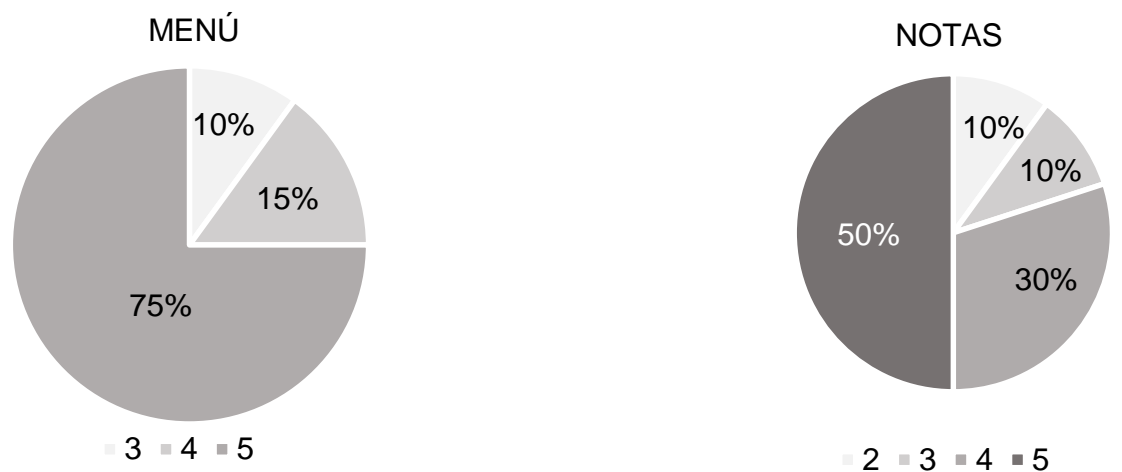

$2-3-4 \backsim 5$

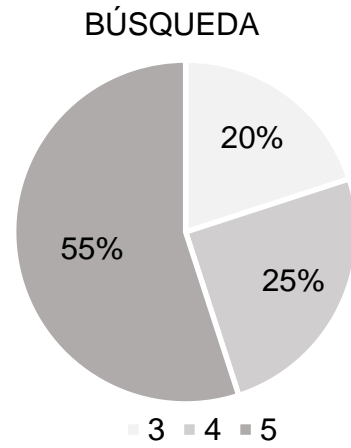

REPOSITORIOS

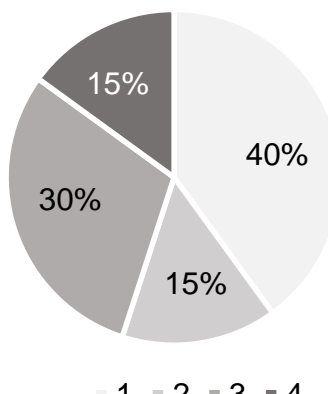

$1=2=3-4$

Fig. 6: Encuesta sobre los paneles de la GUI. Adaptada de (Quiroz, 2017).

En la figura 6 se pueden observar los resultados de la encuesta. De los datos obtenidos se puede observar que la mayoría de los paneles fueron útiles para los participantes exceptuando el panel de repositorios, el cual solo tuvo un $45 \%$ de aceptación; los usuarios expresaron que sería más usable el prototipo si la función del panel de repositorio fuera integrado al panel de contenido.

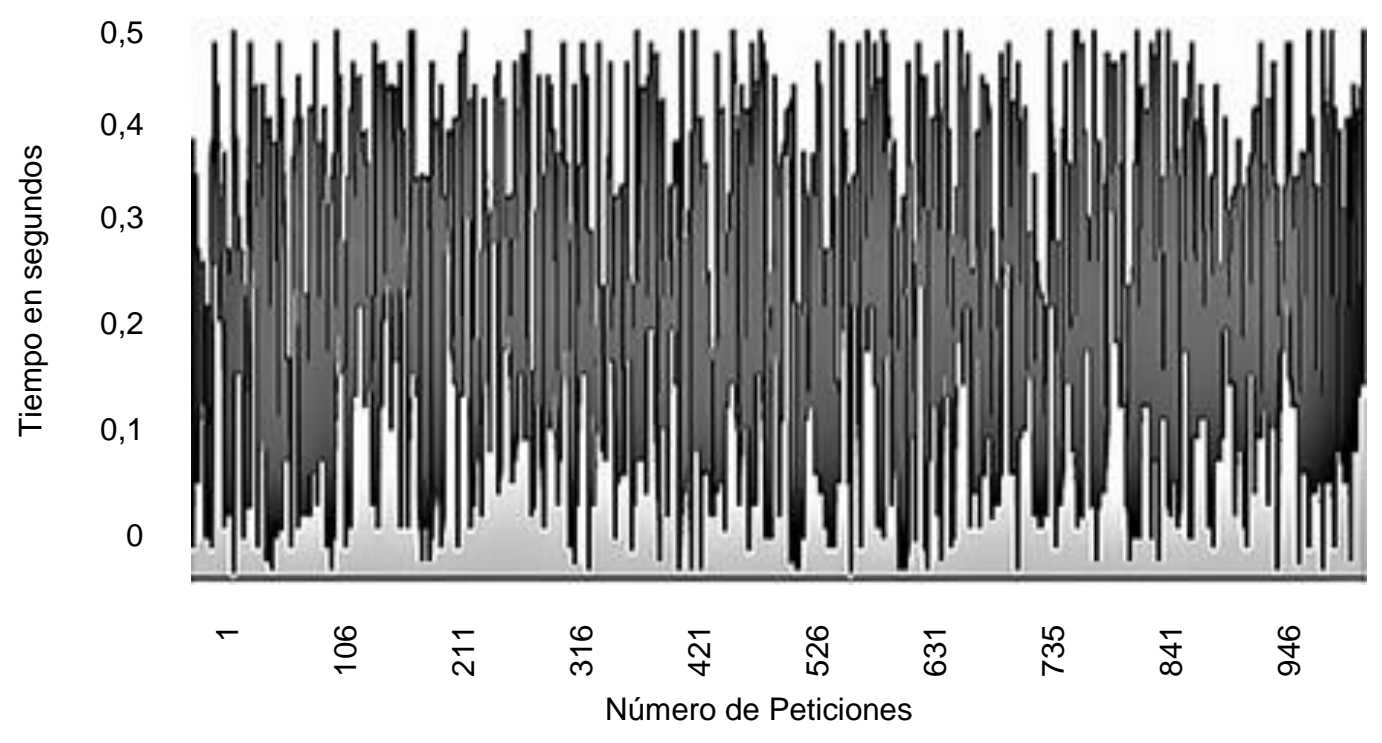

Fig. 7: Tiempos de respuesta vs. peticiones de OAs realizadas. Adaptada de (Quiroz, 2017).

Validación métrica grado de resistencia a la sobrecarga

Para realizar la validación de esta métrica se obtuvo el promedio de respuesta del sistema el cual fue de 0.17 segundos entre OAs con formato video, imagen y PDF, a continuación, se realizó una prueba de estrés de 1000 peticiones. La figura 7 presenta los tiempos de respuesta del sistema al seleccionar un OA. De los datos obtenidos de la prueba de estrés se puede concluir que el grado de resistencia a la sobrecarga del sistema es muy buena, ya que el valor máximo de respuesta es de 0.5 segundos a pesar del gran número de peticiones realizadas. 
Algunos de los resultados obtenidos en el proceso de validación del prototipo implementado son los siguientes: (1) Las recomendaciones inferidas por parte de la ontología para la distribución de la GUI son aceptadas por los usuarios en la mayoría de los casos; (2) Los casos en los cuales los usuarios no aceptan la recomendación de la interfaz adaptativa, se evidenció una reducción en el tiempo para realizar la modificación en la GUl.; (3) Las recomendaciones del SR por los diferentes filtrados (Colaborativo, Conocimiento y contenido) tienen un alto porcentaje de acierto; (4) La visualización y funcionamiento del prototipo en los diferentes navegadores (Chrome, Firefox, Edge y Safari) fue satisfactoria, pues en la mayoría de estos se visualizan los componentes de la GUI correctamente, además de que su funcionamiento es adecuado; (5) La prueba de estrés realizada sobre el sistema, demuestra la capacidad de soportar una gran carga sin un impacto significativo en el tiempo de respuesta de las peticiones.

\section{DISCUSIÓN}

Al comparar el enfoque presentado en este artículo con el de otros investigadores se puede afirmar lo siguiente: este trabajo de investigación incluye mecanismos de adaptación automática de los páneles que componen la interfaz teniendo en cuenta los gustos y preferencias de los usuarios. Adicionalmente, se utiliza el perfil de los usuarios para suministrar recomendaciones sobre el contenido que maneja la interfaz. El trabajo que presenta Park et al. (2007) se basa en menús adaptables, sin embargo, en este enfoque son los usuarios quienes realizan los cambios y no la interfaz en forma automática. Adicionalmente, en este trabajo no se generan recomendaciones sobre el contenido ni se incluyen agentes inteligentes que recomienden contenidos adaptados al perfil del usuario.

Así mismo, aunque el trabajo de Ghédira et al. (2002) propone una interfaz adaptativa basada en el histórico de utilización de la interfaz por parte de dicho usuario para reducir el tiempo de navegación, sin embargo, no se tiene en cuenta otros aspectos del perfil del usuario como intereses y preferencias que puedan permitir una mejor adaptación del contenido presentado. Tampoco utilizan ontologías para representación del conocimiento, ni agentes inteligentes que pudieran agilizar los procesos de adaptación. Adicionalmente, Shakshuki et al. (2015) proponen un SMA con el fin de recolectar información del estado de la salud de los pacientes, sin embargo, los agentes inteligentes son utilizados para cambiar la tonalidad de los colores facilitando de esta forma la visualización del contenido por parte de personas con problemas visuales. Contrastando con nuestro enfoque, no existen aquí otras funcionalidades basadas en el perfil de los usuarios en donde los agentes inteligentes pudieran proponer contenidos interesantes y relevantes para los usuarios.

Finalmente, son pocos los trabajos de investigación que realizan una validación de las interfaces adaptativas propuestas basada en métricas. En el trabajo de Park y Han (2011) se definen cuatro métricas a saber: (1) el tiempo de adaptación del sistema, (2) el tiempo que se demoraron los usuarios para encontrar una opción específica, (3) la eficiencia percibida y (4) las preferencias sobre las interfaces. Nuestra propuesta de validación va más allá y propone seis métricas teniendo en cuenta aspectos más técnicos del sistema, pero analizando el grado de satisfacción del usuario ante las recomendaciones generadas por el sistema, así: a) Rendimiento del sistema; b) Experiencia del usuario con respecto a las recomendaciones de GUI; c) Experiencia del usuario con respecto a las recomendaciones de OAs; d) Comportamiento del prototipo en los navegadores; e) Usabilidad; f) Grado de resistencia a la sobrecarga.

\section{CONCLUSIONES}

El modelo de interfaz adaptativa propuesto aporta elementos de adaptabilidad y adaptatividad al otorgar una flexibilidad al usuario en la visualización de contenidos (cambios en tamaño y posición de los páneles de la GUI) adaptados a su perfil. Adicionalmente, el modelo enfrenta el problema de la sobrecarga de recursos educativos que existen en los repositorios o federaciones de OAs, ya que está en la capacidad de recomendar OAs apropiados, los cuales se adecuan a las características del usuario (i.e. el estilo de aprendizaje).

Teniendo en cuenta los resultados obtenidos, a partir de la validación basada en métricas del prototipo implementado, se demuestra que este tipo de GUI adaptativas son viables y se pueden aplicar a entornos de e-learning, aumentando de esta manera la eficacia de los usuarios en encontrar nuevos recursos que amplíen sus conocimientos. Finalmente, se evidencian las bondades de utilizar agentes inteligentes, ontologías y perfiles de usuario para construir interfaces de usuario adaptativas.

\section{AGRADECIMIENTOS}

La investigación presentada en este artículo fue financiada parcialmente por la beca de investigación de COLCIENCIAS, otorgada a Thomas Quiroz, a través de la Convocatoria Nacional 760 de Jóvenes Investigadores e Innovadores 2015. 


\section{REFERENCIAS}

Acevedo, C.P., J. L. Arciniegas, X. García y J. Perrinet, Proceso de Adaptación de una Aplicación de e-aprendizaje a taprendizaje, Información Tecnológica, 21(6), 27-36 (2010)

Tacy, A., R. Hanson, J. Essington y A. Tokke, GWT in Action, Manning Publications, p. 680 (2013)

Alaoui, S., Y. Idrissi y R. Ajhoun, Building rich user profile based on intentional perspective, Procedia Computer Science, 73, 342-349 (2015)

Berners-Lee, T. y J. Hendler, Publishing on the semantic web, Nature, 1023-1024 (2001)

Felder, R. M. y L.K. Silverman, Learning and teaching styles in engineering education, Engineering Education, 78(7), 674681 (1988)

Ghédira, C., P. Maret, J. Fayn y P. Rubel, Adaptive user interface customization through browsing knowledge capitalization, American Journal of Preventive Medicine, 68, 219-228 (2002)

Jorritsma, W., F. Cnossen y P.M. van Ooijen, Adaptive support for user interface customization: a study in radiology, International Journal of Human-Computer Studies, 77, 1-9 (2015)

Learning Technology Standards Committee, IEEE Standard for Learning Object Metadata, Institute of Electrical and Electronics Engineers, New York (2002)

Letsu-Dake, E. y C.A. Ntuen, A case study of experimental evaluation of adaptive interfaces, International Journal of Industrial Ergonomics, 40(1), 34-40 (2010)

Lhafiane, F., A. Elbyed y M. Bouchoum, Multi-Agent System Architecture Oriented Prometheus Methodology Design for Reverse Logistics, World Academy of Science, Engineering and Technology, Int. Journal of Computer, Electrical, Automation, Control and Information Engineering, 9(8), 1914-1920 (2015)

Liang, Y., H. Zhu, Q. Tian y S. Ji, A method for OWL ontology module partition, Web Society (SWS), IEEE $2^{\text {nd }}$ Symposium on OWL, Beijing, 372 - 377 (2010)

Ovalle, D. A., O.M. Salazar y N.D. Duque, Modelo de Recomendación Personalizada en Cursos Virtuales basado en Computación Ubicua y Agentes Inteligentes, doi: 10.4067/S0718-07642014000600016, Información tecnológica, 25(6), 131-142 (2014)

Park, J., S.H. Han, Y.S. Park e Y. Cho, Adaptable versus adaptive menus on the desktop: Performance and user satisfaction, International Journal of Industrial Ergonomics, 37(8), 675-684 (2007)

Park, J. y S.H. Han, Complementary menus: Combining adaptable and adaptive approaches for menu interface, International Journal of Industrial Ergonomics, 41(3), 305-316 (2011)

Park, D. H., H.K. Kim, I.Y. Choi y J.K. Kim, A literature review and classification of recommender systems research, Expert Systems with Applications, 39(11), 10059-10072 (2012)

Quiroz, T., Modelo de interfaz adaptativa para recomendación de OAs basado en perfiles de usuario, agentes inteligentes y ontologías, Tesis de Maestría en Ingeniería de Sistemas, UNAL, Sede Medellín (2017)

Ravi, R., A. Kumar, K. Bijlani y T.R. Sharika, Self-Adaptive Interface for Comprehensive Authoring, Procedia Computer Science, 58, 158-164 (2015)

Rodríguez, P., N. Duque y D. Ovalle, Método Híbrido de Recomendación Adaptativa de Objetos de Aprendizaje basado en perfiles de Usuario, doi: 10.4067/S0718-50062016000400010, Formación Universitaria, 9(4), 83-94 (2016)

Shakshuki, E.M., M. Reid y T.R. Sheltami, Dynamic Healthcare Interface for Patients, Procedia Computer Science, 63, 356-365 (2015)

Spanoudakis N. y P. Moraitis, Engineering Ambient Intelligence Systems using Agent Technology, IEEE Intelligent Systems, 30(3), 60-67 (2015)

Tudorache, T., N. Csongor, N.F. Noy y M.A. Musen, WebProtégé: A collaborative ontology editor and knowledge acquisition tool for the web, Semantic web, 4(1), 89-99 (2013)

Wang, S.L. y C.Y. Wu, Application of context-aware and personalized recommendation to implement an adaptive ubiquitous learning system, Expert Systems with Applications, 38, 10831-10838 (2011)

Zapata, A., V.H. Menéndez, M.E. Prieto y C. Romero, A framework for recommendation in learning object repositories: An example of application in civil engineering, Advances in Engineering Software, 56, 1-14 (2013)

Zapata, A., V.H. Menéndez, M.E. Prieto y C. Romero, Evaluation and Selection of Group Recommendation Strategies for Collaborative Searching of Learning Objects, International Journal of Human-Computer Studies, 76, 22-39 (2015) 
